\title{
VRML virtual reality technology applied in the mechanical design
}

\author{
Youbing Zhu \\ Chongqing College of Electronic Engineering, Chongqing, 401331, China \\ Email:happylover618@126.com
}

\begin{abstract}
Keywords: Virtual reality construction language, mechanical design, object-oriented, sliding bearing, VRML
\end{abstract}

Abstract. In this paper, VRML2.0 as the development tool to mechanical design of the main parts as the object. First select the typical components of the sliding bearing as the entrance to various forms of three-dimensional model of the structure to user. So that the user has a comprehensive grasp of the structure sliding bearings for the sliding bearing oil pressure distribution, axial movement trajectory. It has a certain analysis. The goal of this paper is to implement a virtual laboratory scenario in which the relevant components of the sliding bearing can be viewed. Secondly, the static pressured distribution characteristics of the simulation. It can be parameterized input the simulate axis of the dynamic load bearing trajectory, in this part only made a qualitative demonstration.

\section{Introduction}

Virtual reality technology uses computers to create a two-dimensional space, by placing the user in the environment, with the help of lightweight multi-dimensional input and output devices [1, 2]. According to the resulting of an immersive to perceive and study the objective world. Users in the virtual environment can "free" movement, free to observe the surrounding scenery [3]. Using two-way data transmission to achieve human-computer interaction, recording the user's actions, and this information as action data sent to the workstation, and then by the action data to modify the model graphics [4]. This action response information is continuously updated and immediately returned to the user's helmet, thus presenting a graphical and audio virtual world synchronized with user actions. In this way, users see a full-color stereo image, the sound is heard in the virtual environment, hands and feet can feel the virtual environment feedback force, resulting in an immersive feel [5].

VMRL from concept to follow-up to the formulation of specifications, have aroused great interest in the relevant industry [6]. HTML presents static text, images, or graphics to users, but VMRL is another new way of presenting data. VMRL virtual reality effect, allowing users to feel like being inside, you can browse the panorama of the Forbidden City, in the library for information, and even shuttle in the museum exhibition room, by virtue of only a computer. However, due to hardware and network transmission constraints, as well as the common considerations between the platform, making the development of VMRL there are some obstacles, which is the future to be overcome problems [7].

\section{Overview of VRML and WorldToolKit}

VRML is a language format based on ASCII code that is used in conjunction with the Internet to describe three-dimensional space. It is the second generation of the Internet to achieve the key technology of virtual reality. The user can interact with the Internet object on the Internet. The basic working principle of VRML can be summarized as: text description, remote transmission, local calculation generation. VRML uses HTML-like textual language to describe 3D scenes. Like programming languages, when a user visits a virtual scene described by VRML, it needs to send the text describing the scene to the local site via the Internet. In general, the text description is embedded in the WEB page, when the browser requests the corresponding page with the page description text to 
send local, and local computing is generated to describe the virtual scene of the data sent to the local, VRML browser into VRML Code files, and interpret it as a graphical image, dynamically generate virtual scenes [8].

The heart of the GTK application is the simulation loop, which calls the wTunivesre-g0 () to enter the simulation loop and the wutnvies_stop () call to exit the simulation loop, as shown in Figure 1.

\section{Perform}

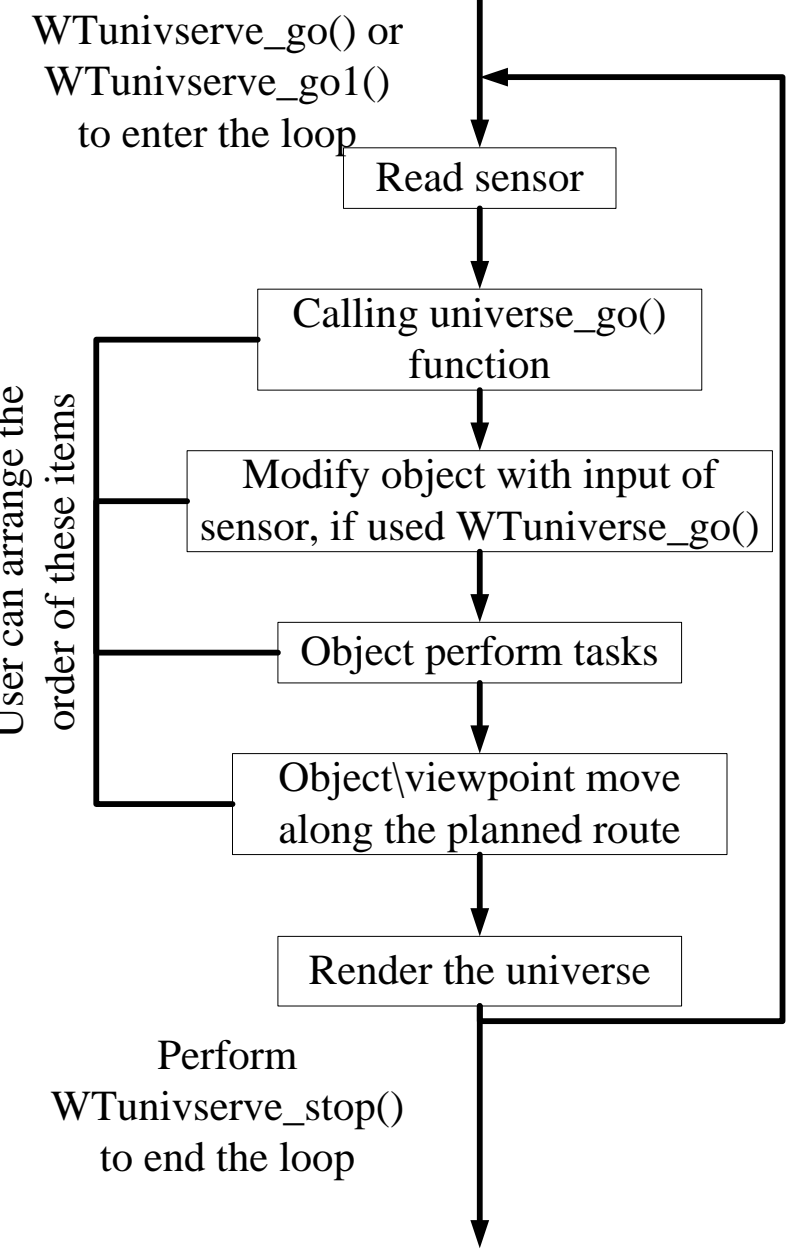

Figure 1. Simulation loop diagram

In a simulation loop, the universe is rendered once per cycle, and the specific way to render the universe is determined directly by the scene graph, which itself changes during program execution. Scene graph is composed of a variety of nodes of the tree diagram, a parent node can have multiple sub-nodes, rendering the scene in the following order and access to the implementation of the corresponding node operation.

\section{Bearing characteristics of radial sliding bearings}

The basic equation of hydrodynamic lubrication theory is the differential equation of fluid membrane pressure distribution. It is derived from the basic equations of viscous hydrodynamics and simplified by the assumptions that the fluid is a Newtonian fluid and that the fluid flow in the fluid film is laminar. The pressure on the viscosity of the fluid Effects of inertia force and gravity. The fluid is considered incompressible; the pressure in the fluid film is constant along the thickness. As shown in Figure 2, the two plates are separated by the lubricating oil. The plate $\mathrm{A}$ is moved in the $\mathrm{x}$-axis direction at the speed $\mathrm{V}$, and the other plate $\mathrm{B}$ is at rest. It is further assumed that the oil does not flow in the z-axis direction between the plates (the dimension of the motion pair in the z-axis direction is infinite). A laminar flow is taken from the oil film to take a micro-unit for analysis. 


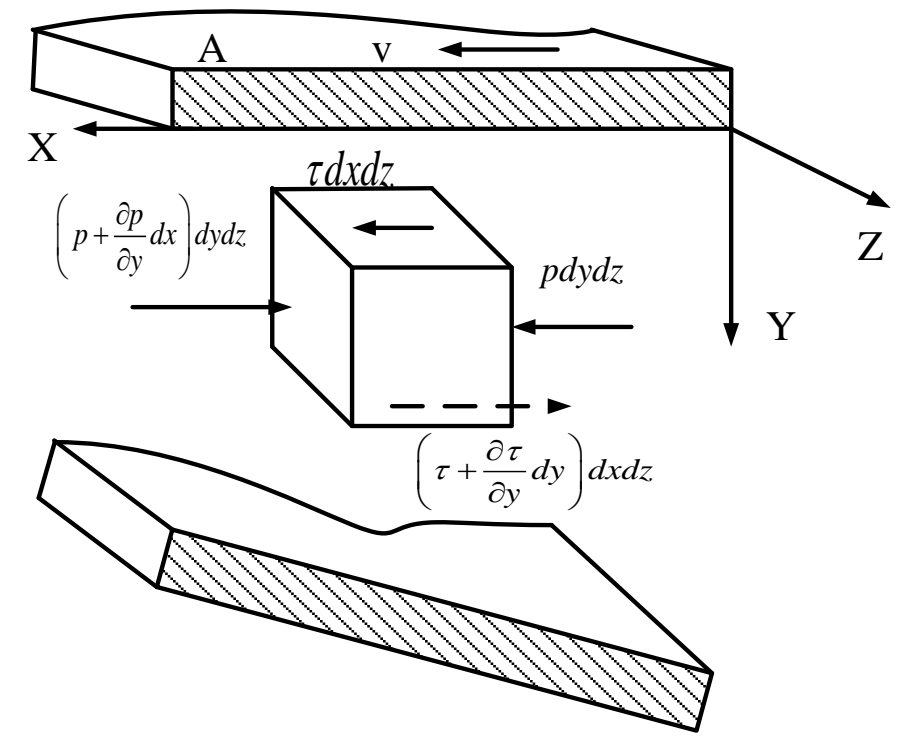

Figure 2. The relative motion of the two plates separated by the oil film

According to the equilibrium condition in $\mathrm{x}$ direction, we get:

$$
p d y d z+\tau d x d z-\left(p+\frac{\partial p}{\partial y} d x\right) d y d z-\left(\tau+\frac{\partial \tau}{\partial y} d y\right) d x d z=0
$$

Newtonian law of viscous fluid friction (that is, anywhere in the fluid shear stress is proportional to the fluid velocity gradient) written in mathematical form:

$$
\begin{gathered}
\tau=-\eta \frac{\partial v}{\partial y} \\
\frac{\partial v}{\partial y^{2}}=\frac{1}{\eta} \frac{\partial p}{\partial x} \\
\frac{\partial v}{\partial y}=\frac{1}{\eta}\left(\frac{\partial p}{\partial x}\right) y+C_{1} \\
v=\frac{1}{2 \eta}\left(\frac{\partial p}{\partial x}\right) y^{2}+C_{1} y+C_{2}
\end{gathered}
$$

\section{Results and discussion}

In reciprocating machines such as internal combustion engines, reciprocating compressors, crank presses and the like, the load acting on the sliding bearing, regardless of size and direction, changes cyclically over time. Such bearings are generally referred to as dynamic bearing. Since the load is variable, the equilibrium positions of the respective instantaneous axes are also changed to form a so-called axis locus. In the dynamic bearing, not only as described above, the load itself has a varying angular velocity, and the journal, the bearing may also have varying angular velocities. In this paper, we discuss the simulation of the axial trajectory of a dynamic load bearing.

When the load on the bearing (size and direction) or angular speed is a constant, such bearings are called steady-state bearings, such as steam turbines. If the load with time and constantly changing, known as the non-steady-state bearings. Although the magnitude and direction of the dynamic or non-steady-state bearing load are variable, the dynamic pressure of the oil film involves the synthesis of the rotation effect and the squeezing effect, but eventually forms a closed axis of change. As the situation of non-steady-state bearing complex, so the axis of the trajectory of the calculation there are many ways. Virtual reality technology applied in the mechanical design was shown in Figure 3. 


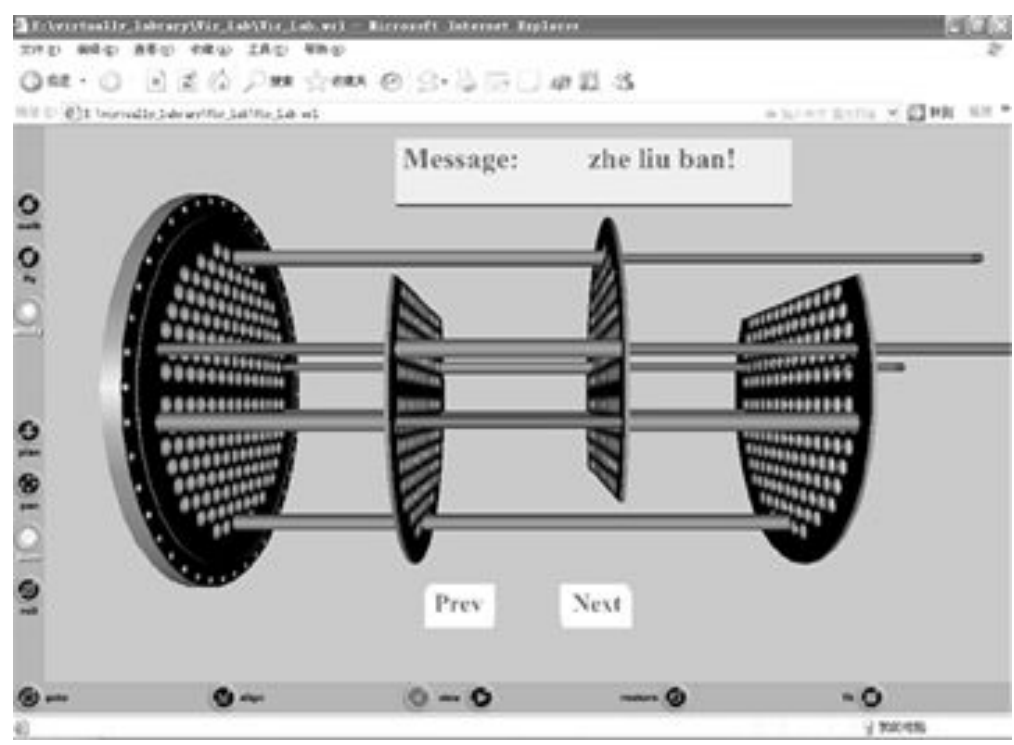

Figure 3. Virtual reality technology applied in the mechanical design

\section{Summary}

In this paper, VRML2.0 is used to develop virtual reality scene based on VRML, which mainly uses windwos98 / NT / 2000 operating system. And the object-oriented software development engineer Visual Basic 6.0 was used to package the whole virtual reality software. The dynamic distribution of the static bearing radial pressure distribution and the axial trajectory of the dynamic load bearing was simulated, and a package was created which could be installed. , Through this package, you can load the relevant auxiliary files and executable programs into your computer system, so you can achieve the proper function of the program.

\section{References}

[1] Handbook of virtual environments: Design, implementation, and applications[M]. CRC Press, 2014.

[2] Sang Y, Zhu Y, Zhao H, et al. Study on an Interactive Truck Crane Simulation Platform Based on Virtual Reality Technology[J]. International Journal of Distance Education Technologies (IJDET), 2016, 14(2): 64-78.

[3] Jiao P G, Shan S F, Miao Q H. Three-Dimensional Virtual Assembly of Automatic Fuel Measuring Device of Diesel Engine Test Bed Based on VRML[C]//Applied Mechanics and Materials. Trans Tech Publications, 2012, 138: 870-873.

[4] Kosmadoudi Z, Lim T, Ritchie J, et al. Engineering design using game-enhanced CAD: The potential to augment the user experience with game elements[J]. Computer-Aided Design, 2013, 45(3): 777-795.

[5] Pepley D, Yovanoff M, Mirkin K, et al. A Virtual Reality Haptic Robotic Simulator for Central Venous Catheterization Training[J]. Journal of Medical Devices, 2016, 10(3): 030937.

[6] Vaughan N, Gabrys B, Dubey V N. An overview of self-adaptive technologies within virtual reality training[J]. Computer Science Review, 2016.

[7] Sang Y, Han Y, Dai Y, et al. The Development of an Interactive Automatic Tool Changer System Based on Virtual Reality Technology[J]. 2016.

[8] Potkonjak V, Gardner M, Callaghan V, et al. Virtual laboratories for education in science, technology, and engineering: A review[J]. Computers \& Education, 2016, 95: 309-327. 